\title{
Correcting Nonuniformities in MRI Intensities Using Entropy Minimization Based on an Elastic Model
}

\author{
Ravi Bansal ${ }^{1,2}$, Lawrence H. Staib ${ }^{3}$, and Bradley S. Peterson ${ }^{1,2}$ \\ 1 Department of Psychiatry, Columbia University, New York, NY 10032 \\ 2 New York State Psychiatric Institute, New York, NY 10032 \\ 3 Departments of Electrical Engineering and Diagnostic Radiology, Yale University, \\ New Haven, CT 06512
}

\begin{abstract}
Nonuniformity in the pixel intensity in homogeneous regions of an observed image is modeled as a multiplicative smooth bias field. The multiplicative bias field tends to increase the entropy of the original image. Thus, the entropy of the observed image is minimized to estimate the original image. The entropy minimization should be constrained such that the estimated image is close to the observed image and the estimated bias field is smooth. To enforce these constraints, the bias field is modeled as a thin-plate deforming elastically. Mathematically, the elastic deformation is described using the partial differential equation (PDE) with the body force evaluated at each pixel. In our formulation, the body force is evaluated such that the overall entropy of the image decreases. In addition, modeling the bias field as an elastic deformation ensures that the estimated image is close to the observed image and that the bias field is smooth. This provides a mathematical formulation which is simple and devoid of weighting parameters for various constraints of interest. The performance of our proposed algorithm is evaluated using both $2 \mathrm{D}$ and $3 \mathrm{D}$ simulated and real subject brain MR images.
\end{abstract}

Keywords: Elastic Deformation, Partial Differential Equation, Expectation Maximization (EM), Entropy

\section{Introduction}

Magnetic resonance imaging (MRI) provides detailed information about anatomical structure. The signal-to-noise ratio (SNR) of the images is improved with the increase in the static magnetic field $\left(B_{0}\right)$ strength. This improved SNR improves the image resolution and the details of the anatomical structures. However, with higher magnetic field strengths, image pixel nonuniformities, i.e. smooth variation in the intensities of the pixels of the same tissue type, tend to increase. This smooth variation of the pixel intensities within a homogeneous region makes automated image processing, such as image segmentation, visualization and derivation of computerized anatomical atlas, more difficult. 
There are various reasons for image nonuniformities [11, which include poor radio frequency $(\mathrm{RF})$ coil uniformity, gradient-driven eddy currents, and subject anatomy both inside and outside the field of view. Some of the causes of nonuniformity, such as frequency response of the receiver and spatial sensitivity of the unloaded RF coils, can be overcome by proper calibration of the MR unit [7]. However, nonuniformities due to the geometry and the magnetic susceptibility of a subject remain [12] and require a post processing approach for correction.

The difficulty of correcting for the intensity nonuniformity stems from the fact that nonuniformities change with MRI acquisition parameters and subjects in the magnet [1]. In addition, the nonuniformities tend to change the tissue statistics such as mean and variance of the pixel intensities 11. Thus, a robust and automatic method for correcting nonuniformities is essential for post-processing MR images. In medical image processing literature, a number of methods have been proposed $\left[\begin{array}{l|l|l|l|l|l|l|l|l|l|l|l}12 & 3 & 6 & 1 & 14 & 2 & 10\end{array}\right]$ with its strengths and weaknesses. Authors in 6] also proposed an entropy minimization method which optimizes a cost function that is a sum of three different terms. Thus, the estimated bias field depends upon the weights of these terms.

We propose a simple and an unifying mathematical framework where the appropriate constraints on the bias field are naturally included. The bias field forms a smooth multiplicative field, a model which has been widely used [12 14. The multiplicative bias field is converted into an additive field by taking the natural logarithm of the pixel intensities of the observed image. Next, the additive bias field is modeled as a thin plate deforming elastically. The elastic deformation is mathematically modeled using the partial differential equations (PDE). This model automatically incorporates the constraints that the bias field should be smooth and the estimated image should be close to the observed image. The body force in the elastic model is evaluated such that the overall entropy of the estimated image is decreased. In evaluating the body force, segmentation of the observed image into tissue classes is required which is achieved using the EM [5] algorithm which iteratively updates the mean and variance of each tissue class.

\section{Methods}

Image nonuniformity is usually modeled as a smooth pixelwise multiplicative bias field 126 . Let $(U(x, y, z))$ denote the bias field and $(T(x, y, z))$ be the pixel intensity of the original image at location $(x, y, z)$, then the observed image pixel intensity $(S(x, y, z))$ is given as $S(x, y, z)=U(x, y, z) \cdot T(x, y, z)$.

The multiplicative bias field is converted into an additive field by taking the natural $\operatorname{logarithm}$ which yields $\ln S=\ln U+\ln T$. Let, $\mathbf{s}, \mathbf{t}$ and $\mathbf{u}$ be the random variables denoting the logarithm pixel intensities sampled from the observed image, the original image and the bias field respectively. Thus, $\mathbf{s}$ is a sum of $\mathbf{t}$ and $\mathbf{u}$. Assuming that the random variables $\mathbf{t}$ and $\mathbf{u}$ are statistically independent, the probability density function of $\mathbf{s}$ is thus obtained by convolving the probability densities of $\mathbf{t}$ and $\mathbf{u}$ [8]. Convolution operator increases entropy and hence the 
entropy of $\mathbf{s}$ is expected to be higher than the entropy of $\mathbf{t}$. Hence, the original image is estimated from the observed image by reducing the entropy of the observed image. In addition, the estimation of the original image is constrained by the assumption that the bias field is smooth and that the original image is close to the observed image.

In our formulation, we model the logarithmic additive bias field as a thin plate deforming elastically under a body force where the body force is evaluated to minimize the entropy of the observed image. In a linear elastic model, the restoring forces are proportional to the displacement. Thus, the elastic model automatically incorporates the constraint that the estimated bias field is smooth and that the estimated image is close to the observed image. The elastic model can be modeled using elliptic partial differential equation (PDE) allowing a simple mathematical formulation which is devoid of various weighing terms. The only weights are $\mu$ and $\lambda$, the viscosity coefficients, which appear in the PDE model of the fluid dynamics. In our implementations, $\mu$ is always set to 1.0 and a variation of $\lambda$ between 0.6 to 1.0 generated good results for most of the images.

The PDE governing the elastic model is given as 4]:

$$
\mu \nabla^{2} \vec{u}+(\lambda+\mu) \vec{\nabla}(\vec{\nabla} \cdot \vec{u})+\vec{b}(\vec{u})=\overrightarrow{0}
$$

where $\nabla^{2}=\nabla^{T} \nabla$ is the Laplacian operator, $(\vec{\nabla} \cdot \vec{u})$ is the divergence operator, $\mu$ and $\lambda$ are the elasticity constant and $\vec{u}(\vec{x}, t)$ is the field at time $t$ and position $\vec{x}$. The PDE describing the elastic model is an elliptic boundary value problem.

The PDE in Eqn. (1), defined on a domain $\Omega=[0,1]^{3}$, is a boundary value problem which is solved numerically using successive over-relaxation (SOR) method with checker board updates $[9$. Zero boundary conditions of $\vec{u}(\vec{x}, t)$ for all $\vec{x} \in \partial \Omega$ and all $t$. Here, $\partial \Omega$ denotes the boundary of the domain $\Omega$. Due to these boundary conditions, the bias field at $\partial \Omega$ will be zero. In our implementation we used Forward Time Centered Space (FTCS) [9] method for numerical estimation of the various partial derivatives in Eqn. (1).

To evaluate the body force at a pixel, the image pixels are first classified into different classes using EM [5] based maximum likelihood classification algorithm using the current estimated bias field. For the estimated classification of each pixel, body force is determined which is used to update the bias field. Using the estimated bias field, the pixel classification is again updated using the EM algorithm. This process is repeated till the estimated pixel classifications converge.

\subsection{EM Based Segmentation}

We assume that the image pixels can be labeled into 4 classes \{background, gray matter, white matter, fat\}. The pixel intensities in these classes are assumed to be Gaussian distributed. Thus, we need to estimate the mean and variance of the pixel intensities in each of these classes and the class to which a pixel belongs. For a given current estimate of the bias field, we estimate the class mean and variance and the pixel classification using the EM algorithm [5]. 
Let, $\mu=\left(\mu_{1}, \mu_{2}, \ldots, \mu_{n}\right)$ denote the means, $\sigma=\left(\sigma_{1}, \sigma_{2}, \ldots, \sigma_{n}\right)$ denote the standard deviations and $\pi=\left(\pi_{1}, \pi_{2}, \ldots, \pi_{n}\right)$ denote the prior probabilities of the $n$ classes with constraint that $\sum_{i} \pi_{i}=1$. Let, the class labels be $c_{1}, c_{2}, \cdots, c_{n}$ and let $z_{i}=\left(z_{1 i}, z_{2 i}, \ldots, z_{n i}\right)$ be a vector of size $n$ in $\{0,1\}$ such that $z_{i}=$ $(0,0, \ldots, 0, \underbrace{1}_{m}, 0, \ldots, 0)$ if the $i$ th pixel belongs to $m$ th class. That is, the vector $z_{i}$ is all zero except for a 1 at the $m$ th location if the pixel belongs to the $m$ th class. Then given the observed image $S$ and the estimated bias field $U$, the likelihood probability $P\left(s_{i} \mid u_{i}, \mu, \sigma\right)$ of the observed data at the $i$ th pixel is given as:

$$
P\left(s_{i} \mid u_{i}, \mu, \sigma\right)=\sum_{b=1}^{n} \pi_{b} p_{b}\left(s_{i} \mid u_{i}, \mu_{b}, \sigma_{b}\right)=\prod_{a=1}^{n}\left[\sum_{b=1}^{n} \pi_{b} p_{b}\left(s_{i} \mid u_{i}, \mu_{b}, \sigma_{b}\right)\right]^{z_{a i}} .
$$

Then, $P\left(c_{i} \mid s_{i}, u_{i}, \sigma, \mu\right)=\frac{P\left(s_{i}, c_{i} \mid u_{i}, \mu, \sigma\right)}{P\left(s_{i} \mid u_{i}, \mu, \sigma\right)}=\prod_{d}\left[\frac{\pi_{a} p_{a}\left(s_{i} \mid u_{i}, \mu_{a}, \sigma_{a}\right)}{\sum_{b=1}^{n} \pi_{b} p_{b}\left(s_{i} \mid u_{i}, \mu_{b}, \sigma_{b}\right)}\right]^{z_{d i}}$.

Thus, for the whole image, assuming that the image pixel intensities are sampled independently, the maximum likelihood estimates of the class mean and variances using the EM algorithm are given as:

$$
\mu_{a}=\frac{\sum_{i} \widehat{z_{a i}}\left(s_{i}-u_{i}\right)}{\sum_{j} \widehat{z_{a j}}}, \sigma_{a}^{2}=\frac{\sum_{i} \widehat{z_{a i}}\left(s_{i}-u_{i}-\mu_{a}\right)^{2}}{\sum_{j} \widehat{z_{a j}}}, \widehat{z_{a i}}=\frac{\pi_{a} p_{a}\left(s_{i} \mid u_{i}, \mu_{a}, \sigma_{a}\right)}{\sum_{b=1}^{n} \pi_{b} p_{b}\left(s_{i} \mid u_{i}, \mu_{b}, \sigma_{b}\right)},
$$

where $\widehat{z_{a i}}$ denotes the expected value of the $i$ th pixel belonging to class $a$. Thus, the EM algorithm, in addition to estimating the class variables, also estimates a probabilistic segmentation of the image.

\subsection{The Body Force}

For a given estimate of the pixel classification and an estimated bias field, a body force at each pixel is estimated by minimizing the joint conditional entropy $H(C, T \mid S, U)$ which is a measure of uncertainty in the estimated classification and the bias field corrected image for a given observed image and the current estimated bias field. To evaluate this entropy, consider the probability distribution $p(C, T \mid S, U)$ which is simplified, assuming that the neighboring pixels in the observed image are statistically independent, as $p(C, T \mid S, U)=\prod_{i} p\left(c_{i}, t_{i} \mid s_{i}, u_{i}\right)=$ $\prod_{i} P\left(c_{i}=a\right) p_{a}\left(t_{i} \mid s_{i}, u_{i}\right)$

Thus, the joint conditional entropy is evaluated as:

$$
\begin{aligned}
H(C, T \mid S, U) & =-\sum_{C, T} p(C, T \mid S, U) \log p(C, T \mid S, U) \\
& =-\sum_{i}\left(\sum_{a, t_{i}} p\left(c_{i}=a, t_{i} \mid s_{i}, u_{i}\right) \log p\left(c_{i}=a, t_{i} \mid s_{i}, u_{i}\right)\right)=\sum_{i} H\left(c_{i}, t_{i} \mid s_{i}, u_{i}\right),
\end{aligned}
$$

where the entropy $H\left(c_{i}, t_{i} \mid s_{i}, u_{i}\right)$ is given as:

$$
H\left(c_{i}, t_{i} \mid s_{i}, u_{i}\right)=-\sum_{a} P\left(c_{i}=a\right) \log p\left(c_{i}=a\right)-\sum_{a, t_{i}} P\left(c_{i}=a\right) p_{a}\left(t_{i} \mid s_{i}, u_{i}\right) \log p_{a}\left(t_{i} \mid s_{i}, u_{i}\right) .
$$


Since the pixel classifications remain constant while estimating the bias field, the body force is evaluated which minimizes $H(C, T \mid S, U)$, i.e.,

$$
\min _{T} H(C, T \mid S, U)=\min _{T} \sum_{i} H\left(c_{i}, t_{i} \mid s_{i}, u_{i}\right)=\min _{T} \sum_{a} H_{a}(t \mid s, u) \frac{\sum_{i} P\left(c_{i}=a\right)}{N} .
$$

To evaluate the entropy functions $H_{a}(t \mid s, u)$, the probability density functions $p_{a}(t \mid s, u)$ are estimated using the Gaussian kernel based Parzen window estimate [13, i.e.,

$$
p_{a}(t \mid s, u)=\frac{1}{\sum_{i} P\left(c_{i}=a\right)} \sum_{i}^{N} P\left(c_{i}=a\right) \frac{1}{\sigma_{a} \sqrt{2 \pi}} \exp \left[-\frac{1}{2} \frac{\left(t-t_{i}\right)^{2}}{\sigma_{a}^{2}}\right],
$$

where $t=s-u$ and $t_{i}=s_{i}-u_{i}$. For heuristic computation of the entropy $H_{a}(t \mid s, u)$, consider two samples of pixels $\left(t_{i}, t_{j}\right), \forall i, j$ selected at randomly from the current estimated image. The entropy terms, $H_{a}(t \mid s, u)$, are evaluated as:

$$
H_{a}(t \mid s, u)=\ln \left(\sigma_{a} \sqrt{2 \pi} \sum_{i} P_{i}(a)\right)-\frac{\sum_{j} P_{j}(a)\left\{\ln \sum_{i=1}^{N} P_{i}(a) \exp \left[-\frac{1}{2} \frac{\left(t_{j}-t_{i}\right)^{2}}{\sigma_{a}^{2}}\right]\right\}}{\sum_{j=1}^{M} P_{j}(a)} .
$$

To evaluate $\min _{T} H_{a}(t \mid s, u)$, we will evaluate variation in $H_{a}(t \mid s, u)$ for small change in the bias field. Let, $\Delta \vec{u}(\vec{x}, t)$ denote the small variation in the bias field at a pixel location $\vec{x}$ at time $t$. Also, let $h=\max \vec{x}\|\Delta \vec{u}(\vec{x}, t)\|$, be the maximum variation in the bias field over all pixel locations. Then the variation in the entropy for small change in the bias field is evaluated as follows:

$$
\begin{aligned}
\frac{d H_{a}(t \mid s, u)}{d \vec{u}(\vec{x}, t)} & =\operatorname{lt}_{h \rightarrow 0}\left\{\frac{H_{a}(t \mid s, u+\Delta u)-H_{a}(t \mid s, u)}{h}\right\} \\
& =\frac{1}{\sigma_{a}^{2} \sum_{j=1}^{M} P_{j}(a)} \sum_{j} P_{j}(a)\left\{\sum_{i=1}^{N} W_{a}(i, j)\left(t_{j}-t_{i}\right) \frac{\partial}{\partial h}\left(t_{j}-t_{i}\right)\right\} \\
& \text { where, } \quad W_{a}(i, j)=\frac{P_{i}(a) \exp \left(\frac{-1}{2}\left(\frac{t_{j}(h)-t_{i}(h)}{\sigma_{a}}\right)^{2}\right)}{\sum_{k} P_{k}(a) \exp \left(\frac{-1}{2}\left(\frac{t_{j}(h)-t_{k}(h)}{\sigma_{a}}\right)^{2}\right)} .
\end{aligned}
$$

To evaluate $\frac{\partial}{\partial h}\left(t_{j}-t_{i}\right)$, consider a second set of samples $\left(t_{i}^{\prime}, t_{j}^{\prime}\right) \forall i, j$ selected at random from the estimated image with the small variation $\Delta \vec{u}(\vec{x}, t)$ in the bias field. Let $d_{j}= \pm$ denote increase or decrease in the pixel intensity at the pixel $j$ in the template image and let $d_{i}= \pm$ be the increase or decrease at the pixel $i$. Also, let the image gradient at the pixel $j$ be denoted as $\nabla T_{j}=\left(\frac{\partial t_{j}}{\partial x^{(1)}} \frac{\partial t_{j}}{\partial x^{(2)}} \frac{\partial t_{j}}{\partial x^{(3)}}\right)^{T}$. Then the partial derivatives $\frac{\partial t_{j}}{\partial h}$ are defined as $\frac{\partial t_{j}}{\partial h}=\operatorname{lt}_{h \rightarrow 0}\left(\frac{t_{j}^{\prime}-t_{j}}{h}\right)=\left\|\nabla T_{j}\right\| d_{j}$, which is the magnitude of the gradient in the deformed template image multiplied with the direction of bias field variation at the voxel $j$. Similarly, the partial derivative $\frac{\partial t_{i}}{\partial h}$ is evaluated as $\frac{\partial t_{i}}{\partial h}=\left\|\nabla T_{i}\right\| d_{i}$.

Let $s_{i j}$ be the sign which between $d_{i}$ and $d_{j}$, i.e., $d_{i}=s_{i j} d_{j}$. Thus, using these, we have $\frac{\partial}{\partial h}\left(t_{j}-t_{i}\right)=\frac{\partial t_{j}}{\partial h}-\frac{\partial t_{i}}{\partial h}=\left(\left\|\nabla T_{j}\right\|-s_{i j}\left\|\nabla T_{i}\right\|\right) d_{j}$. 


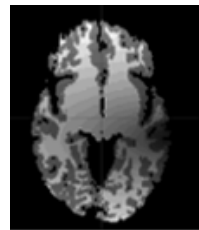

(a)

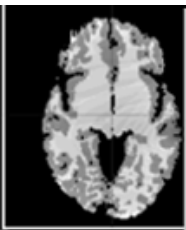

(b)

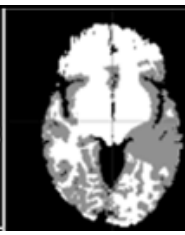

(c)

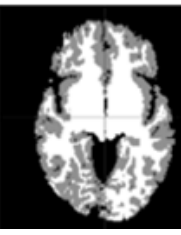

(d)

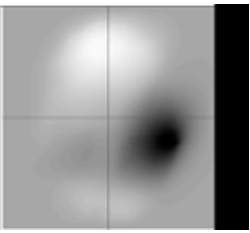

(e)

Fig. 1. Synthetic data with simulated bias field. (a) Original MRI. (b) Nonuniformity corrected. (c) Segmentation of (a). (d) Segmentation of (b). (e) Estimated bias field.

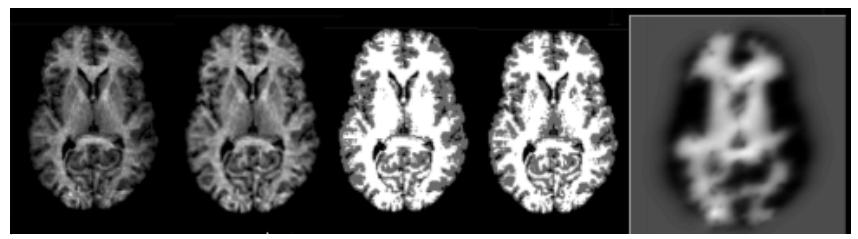

(a) (b)

(c)

(d)

(e)

Fig. 2. Real subject 2D MR image. (a) Original MRI. (b) Nonuniformity corrected. (c) Segmentation of (a). (d) Segmentation of (b). (e) Estimated bias field.

Hence, the partial derivative of the marginal entropy can be written as:

$$
\frac{d H_{a}(t \mid s, u)}{d h}=\frac{1}{\sum_{j} P_{j}(a)} \sum_{j} \underbrace{P_{j}(a)\left\{\frac{1}{\sigma_{a}^{2}} \sum_{i} W_{a}(i, j)\left(t_{j}-t_{i}\right)\left(\left\|\nabla T_{j}\right\|-s_{i j}\left\|\nabla T_{i}\right\|\right)\right\}}_{b_{j}(a)} d_{j} .
$$

Thus, we use the body force at the $j$ pixel due to the class $a$ is given as

$$
b_{j}(a)=P_{j}(a)\left\{\frac{1}{\sigma_{a}^{2}} \sum_{i} W_{a}(i, j)\left(t_{j}-t_{i}\right)\left(\left\|\nabla T_{j}\right\|-s_{i j}\left\|\nabla T_{i}\right\|\right)\right\} .
$$

And the total body force at the pixel $j$ is then given by the sum, $b_{j}=$ $\sum_{a} \frac{P_{i}(a)}{N} b_{j}(a)$.

\section{Results}

In this section we present results of our intensity nonuniformity correction algorithm using both real and synthetic 2D and 3D MR image data.

Fig. 1 (a), shows a synthetic image with added known bias field. This synthetic image is obtained by first manually segmenting a 2D MR image into gray and white matter and then adding an off-center parabolic bias field. In this synthetic image, the gray matter pixel intensities vary from 38 to 58 and the white matter intensities vary from 46 to 57 . Thus, there is a substantial overlap of pixel intensities. Fig. 1 (b) shows the image nonuniformity corrected image obtained 
using our proposed algorithm. In this image, the gray matter pixel intensity variation is from 46 to 58 and the white matter pixel intensity variation is from 56 to 67 . Hence, in the corrected image, the variation of the pixel intensities within a class is reduced, the intensity overlap of the two classes is greatly reduced.

Fig. 1 (c) and (d) show the EM based segmentation of the images in Fig. 1 (a) and (b) respectively. Note that the segmentation is greatly improved after correcting for intensity nonuniformities. Numerically, for the image in Fig. [1 (c), the mis-classification rates of the EM algorithm are $24 \%$ and $12 \%$ for the gray matter and the white matter respectively. However, after correction, for the image in Fig. 1 (d), the mis-classification rates are $0 \%$ and $0.05 \%$ for the gray and white matter respectively.

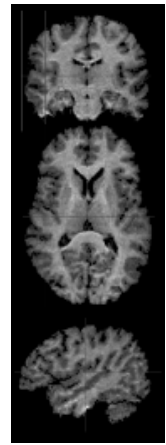

(a)

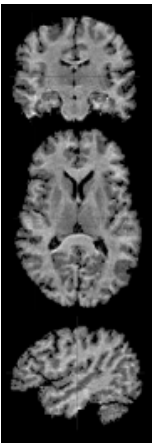

(b)

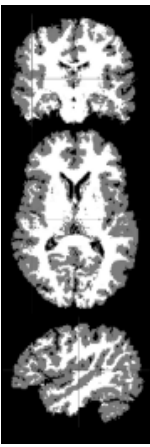

(c)

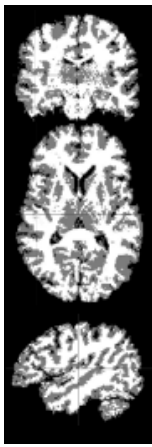

(d)

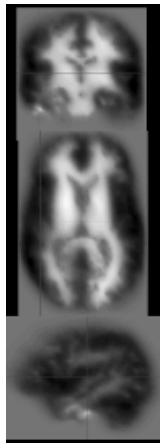

(e)

Fig. 3. Real subject 3D MRI. (1) Original MRI. (b) Nonuniformity corrected. (c) estimated segmentation of (a). (d) Estimated segmentation of (b). (e) Estimated bias field.

Fig. 2 (a) shows a real subject 2D MR image with nonuniformities. The image corrected for the nonuniformities using our algorithm is shown in Fig. 2 (b). Note that the corrected image has much better gray matter to white matter contrast. The estimated bias field is shown in Fig. 2 (e). Fig. 2 (c) and (d) show the EM based segmentation of the images in Fig. 2 (a) and (b) respectively.

Fig. 3 (a) shows three orthogonal views of a real subject 3D MR of a subject. Image obtained after correcting for intensity nonuniformities is shown in Fig. 3 (b). Fig. 3 (c) and (d) show the EM based segmentation of the images in Fig. 3 (a) and (b) respectively.

These results show that the nonuniformities in the pixel intensities can be corrected by minimizing overall entropy of the image. Also, our proposed method is robust and automated as there are no weighing parameters to be set. The estimated segmentation of the final intensity corrected image shows nonuniformity correction can lead to better post-processing of the acquired images. Also, since no underlying model of the deformation field is being assumed, the method can be used to correct any smooth bias field. 


\section{Discussion}

We present a mathematical framework for correcting intensity nonuniformities where the bias field is modeled as a thin plate deforming elastically under a body force. The body force at each pixel is evaluated such that the overall entropy of the estimated image is reduced. The elastic deformations of the intensities is modeled using the partial differential equation (PDE), which is solved using the successive over relaxation (SOR) method.

Modeling the bias field as elastic deformations has the following advantages. First, it ensures that the estimated image is close to the original image. This is due to the fact that in the linear elastic model, the restoration forces are proportional to the amount of deformation. Second, the smoothness of the estimated bias field is ensured by the PDE modeling the elastic model. Third, the model is free of weighing parameters.

\section{References}

[1] Ahmed, M., Yamany, S., Mohamed, N., and Farag, A. (1999). A modified fuzzy Cmeans algorithm for MRI bias field estimation and adaptive segmentation. $M I C$ CAI, pages $72-81$.

[2] Arnold, J., Liow, J., Schaper, K., Stern, J., Sled, J., Shattuck, D., Worth, A., Cohen, M., Leahy, R., Mazziotta, J., and Rottenberg, D. (2001). Qualitative and quantitative evaluation of six algorithms for correcting intensity nonuniformity effects. NeuroImage, 13:931-943.

[3] Cohen, M., DuBois, R., and Zeineh, M. (2000). Rapid and effective correction of RF inhomogeneity for high field magnetic resonance imaging. Human Brain Mapping, 10:204-211.

[4] Davatzikos, C. et al. (1996). A computerized method for morphological analysis of the corpus callosum. J. Comp. Assis. Tomo., 20:88-97.

[5] Dempster, A. P., Laird, N. M., and Rubin, D. B. (1977). Maximum likelihood from incomplete data via EM algorithm. J. Royal Statistical Soc., Ser. B, 39:1-38.

[6] Mangin, J. (2000). Entropy minimization for automatic correction of intensity nonuniformity. IEEE Workshop on Math. Methods in Bio. Image Analysis (MM$B I A)$, pages $162-169$.

[7] McVeigh, E., Bronskill, M., and Henkelman, R. (1986). Phase and sensitivity of receiver coils in magnetic resonance imaging. Med. Phys., 13:806-814.

[8] Papoulis, A. (1991). Probability, Random Variable, and Stochastic Processes. McGraw-Hill, Inc., 3 edition.

[9] Press, W. H., Teukolsky, S. A., Vetterling, W. T., and Flannery, B. P. (1992). Numerical Recipes in C. The Art of Scientific Computing. Cambridge University Press.

[10] Prima, S., Ayache, N., Barrick, T., and Roberts, N. (2001). Maximum likelihood estimation of the bias field in mr brain images: Investigating different modelings of the imaging process. Medical Image Computing and Computer-Assisted Intervention (MICCAI'01), LNCS 2208:811-819.

[11] Simmons, A., Tofts, P., Barker, G., and Arridge, S. (1994). Sources of intensity nonuniformity in spin echo images. Magn. Reson. Med., 32:121-128. 
[12] Sled, G., Zijdenbos, A., and Evans, A. (1998). A nonparametric method for automatic correction of intensity nonuniformity in MRI data. IEEE Trans. of Medical Imaging, 17(1):87-97.

[13] Viola, P. and Wells, W. M. (1995). Alignment by maximization of mutual information. Fifth Int. Conf. on Computer Vision, pages 16-23.

[14] Wells, W., Grimson, W., and Kikinis, R. (1996). Adaptive segmentation of MRI data. IEEE Trans. on Medical Imaging, 15(4):429-442. 